\title{
STIMULATORY CAPACITY OF AN OCTACALCIUM PHOSPHATE/GELATIN COMPOSITE ON BONE REGENERATION IN A RABBIT TIBIA DEFECT MODEL
}

\author{
Kentaro Suzuki ${ }^{1,2}$, Yoshitomo Honda ${ }^{2}$, Takahisa Anada ${ }^{2}$, Takuto Handa ${ }^{3,4}$, Naohisa Miyatake ${ }^{2,5}$, \\ Atsushi Takahashi ${ }^{1}$, Masami Hosaka ${ }^{1}$, Hideki Imaizumi ${ }^{2,6}$, Eiji Itoi ${ }^{1}$, and Osamu Suzuki ${ }^{2} *$ \\ (*Corresponding author: suzuki-o@m.tohoku.ac.jp) \\ ${ }^{1}$ Department of Orthopaedic Surgery, Graduate School of Medicine, Tohoku University, \\ 2-1 Seiryo-machi, Aoba-ku, Sendai 980-8575, Japan. \\ ${ }^{2}$ Division of Craniofacial Function Engineering, Tohoku University Graduate School of Dentistry, \\ 4-1 Seiryo-machi, Aoba-ku, Sendai 980-8575, Japan. \\ ${ }^{3}$ Shinoda General Hospital, 2-68, Sakura-cho, Yamagata 990-0045, Japan. \\ ${ }^{4}$ Division of Oral Surgery, Tohoku University Graduate School of Dentistry, 4-1 Seiryo-machi, Aoba-ku, Sendai \\ 980-8575, Japan. \\ ${ }^{5}$ Sendai Orthopaedic Hospital, 24 Izai aza Higashi-tori, Wakabayashi-ku, Sendai 984-0038, Japan. \\ ${ }^{6}$ Osaki Citizen Hospital, 2-3-10 Senjuji-machi, Furukawa, Osaki 989-6183, Japan.
}

Key words: Octacalcium phosphate, Gelatin, Bone regeneration, Long bone, Tibia

\begin{abstract}
The present study was designed to investigate whether an octacalcium phosphate/gelatin (OCP/Gel) composite can repair a defect created in rabbit tibia within 4 weeks. Histological and $\mu \mathrm{CT}$ studies showed that the implantation of OCP/Gel composite (40 wt\% OCP) enhances cortical bone deposition over the defect accompanied by the formation of cartilage and bone marrow with cancellous bone within 2 weeks. The defects were repaired even in the control group (defect only) within 4 weeks; however, the repaired cortical bone was thinner than that in the OCP/Gel-implanted group. The results suggest that an OCP/Gel composite could be effectively used as a bone substitute material for autografts.
\end{abstract}

(Received February 21, 2012; Accepted March 26, 2012)

\section{INTRODUCTION}

Octacalcium phosphate (OCP) crystals, having specific material characteristics, have been recognized to display a stimulatory capacity on osteoblast differentiation from stromal cells ${ }^{1,2}$ and osteoclast formation from the precursor cells in co-culturing with osteoblasts ${ }^{3}$ in vitro. In fact, OCP and OCP-based materials with natural polymers, such as $\mathrm{OCP} /$ collagen composite, enhance bone regeneration coupled with their own biodegradation in various experimentally created bone defects. ${ }^{4-8}$ From the viewpoint of bone regenerative properties, autologous bone is still the first choice for filling defects due to its superior osteoconductivity, since autologous bone consists of osteoblastic cells, growth factors, such as bone morphogenetic proteins, and matrix materials, including collagen and hydroxyapatite (HA) crystals. Although the tissue-engineering technique by introducing exogenous cells into the OCP-based material certainly increases the osteogenic capability of the material, ${ }^{9}$ the use of bone-substitute materials that have biological activity compatible with that of autologous bone, with the stimulation of host osteoblastic cells around OCP crystals, is still preferable from the viewpoint of reducing the complex handling and the cost of the preparation of an implant form. We recently succeeded in synthesizing such material ${ }^{10}$ with bone regenerative capability approaching that of autologous bone to repair a critically sized rat calvaria defect composed of an OCP co-precipitated gelatin (Gel) composite obtained through OCP crystal growth in a solution containing Gel molecules. However, the effect of $\mathrm{OCP} / \mathrm{Gel}$ composite on bone regeneration in long bone defect has never been examined. In the present study, the bone regenerative capacity of an $\mathrm{OCP} / \mathrm{Gel}$ composite containing $40 \mathrm{wt} \%$ of OCP was examined for a bone defect experimentally created in rabbit long bone. The OCP/Gel with $40 \mathrm{wt} \%$ of OCP is a composite that repairs most the intramembranous bone regeneration of the rat critical sized calvaria bone defect in a comparative study using various $\mathrm{OCP} / \mathrm{Gel}$ composites containing 24 to $40 \mathrm{wt} \%$ of $\mathrm{OCP}^{10)}$

\section{MATERIALS AND METHODS}

1) Preparation of implants

The direct precipitation from a supersaturated 
calcium and phosphate solution was performed in a hot solution in the presence of Gel molecules according to methods previously reported. ${ }^{4,10}$ Briefly, the reagent grade of $\mathrm{Gel}$ isolated from porcine skin with an acidic process (Sigma-Aldrich, St. Louis, MO) was used. A calcium solution was mixed with a phosphate solution to attain supersaturation with respect to OCP to enhance the precipitation of OCP crystals in the presence of $0.5 \mathrm{wt} \%$ of Gel molecules for the total volume of the calcium and phosphate solutions. OCP alone was also prepared ${ }^{4}$ for the comparative purpose of material characterization. In addition, gelatin without OCP from a concentration corresponding to the $\mathrm{OCP} / \mathrm{Gel}$ preparation was obtained. The OCP/Gel precipitate or gelatin was recovered, and then lyophilized. The disks of OCP/Gel were molded $(5 \mathrm{~mm}$ in diameter, $4 \mathrm{~mm}$ in thickness) and subjected to a dehydrothermal treatment in a vacuum drying oven. The lyophilized OCP/Gel composite, gelatin, and OCP were characterized by X-ray diffraction with $30 \mathrm{KV}$ and 15 $\mathrm{mA}$ in the $2 \theta$ range from 3 to 60 degrees (XRD, MiniFlex, Rigaku Electrical, Tokyo, Japan) and Fourier transform infrared spectroscopy (FTIR, FREEXACT-2, Horiba, Kyoto, Japan) in the wavelength range of $400-2000 \mathrm{~cm}^{-1}$ with $4 \mathrm{~cm}^{-1}$ resolution.
A

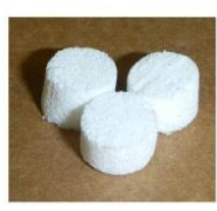

B

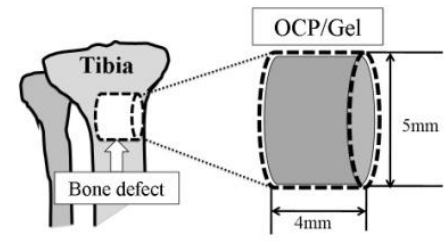

FIGURE 1. Photograph of OCP/Gel (A) and schematic view of OCP implantation with an experimental bone defect model in rabbit tibia (B).

2) Surgical procedure and tissue preparation
A rabbit tibia defect model previously reported ${ }^{11}$ was referenced. Eight male Japanese white rabbits weighing $2.8-3.2 \mathrm{~kg}$ were used. The principles of laboratory animal care and use and national laws were followed. All procedures were approved by the Animal Research Committee of Tohoku University. The rabbits were anesthetized with intravascular injection of ketamine hydrochloride $(0.5 \mathrm{mg} / \mathrm{kg})$. The skin and periosteum of the tibia were dissected and then raised from the bone surface. A periosteal bone defect $(5 \mathrm{~mm}$ in diameter) was created by using trephine drill. An OCP/Gel disk was implanted into the bone defect. The untreated group (defect only) was used in the control experiment without the implants. Figure 1 shows the OCP/Gel composites obtained (Fig. 1A) and the implantation using the experimental bone defect model in rabbit tibia (Fig. 1B). At 2 and 4 weeks, the experimental rabbits were sacrificed, and the tibia bone, including the implant, was retrieved. After the tissue was analyzed using a Microfocus X-Ray CT System with $90 \mathrm{KV}, 110 \mu \mathrm{A}$ (Scan Xmate-E090, Comscan Techno Co., Ltd., Kanagawa, Japan), the tissue was fixed in $4 \%$ paraformaldehyde, decalcified with 10\% EDTA, and embedded in paraffin. Four- $\mu \mathrm{m}$-thick serial sections were prepared coronally and stained with hematoxylin and eosin (HE).

\section{RESULTS AND DISCUSSION}

1) Characteristics of $\mathrm{OCP} / \mathrm{Gel}$ composite, $\mathrm{OCP}$, and Gel

The OCP/Gel composite obtained was molded into various shapes, including the present rod-like form. As shown in Figure 1A, the OCP/Gel composite was a spongy material that was readily implanted into the various defects, ${ }^{10}$ including the present long bone defect. Figure 2 shows XRD patterns (A) and FTIR spectra (B) of OCP, OCP/Gel, and gelatin. The XRD patterns obtained from the control OCP and OCP/Gel contained the

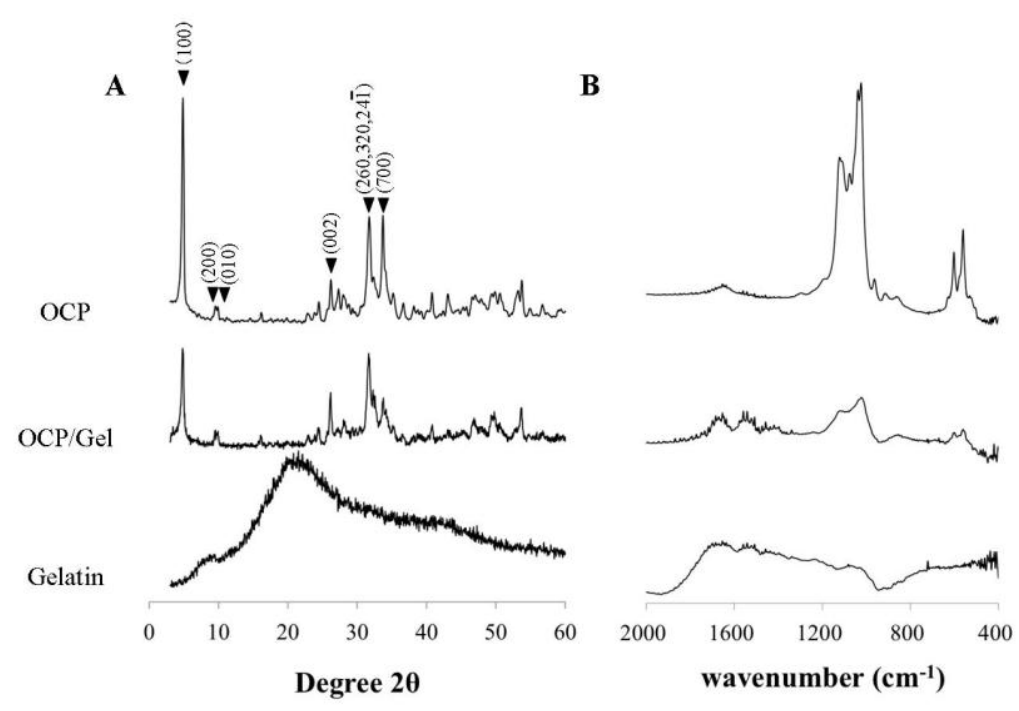

FIGURE 2. XRD patterns (A) and FTIR spectra (B) of OCP, OCP/Gel, and gelatin. 
characteristic (100) reflection at $2 \theta=4.8^{\circ}$ and the (010) peak at $2 \theta=9.8^{\circ}$, which corresponded well to those expected from the OCP structure $^{12}$ (Fig. 2A). The results confirmed that the crystals grown within the Gel matrix had a single OCP phase. In the FTIR spectra of OCP, OCP/Gel, and gelatin, amide bands were still detected between 1300 and $1800 \mathrm{~cm}^{-1}$ in the spectrum of OCP/Gel, which was derived from the gelatin in the composite. Phosphate vibration bands in 500-650 and $900-1250 \mathrm{~cm}^{-1}$ of OCP were obscure in OCP/Gel. The XRD pattern of OCP/Gel was broad in comparison with that of OCP. The results suggest that the OCP crystals were embedded within the gel matrix and that the precipitation contained a very small amount of amorphous calcium phosphate
(ACP). In fact, the previous study with transmission electron microscopy showed that small sphere-like particles were involved adjacent to large plate-like OCP crystals at the ultrastructural level. ${ }^{10}$

\section{2) Micro-CT evaluation}

The micro-CT images of the treated regions in the tibia are shown in Figure 3. The present conditions used for measurement do not exhibit any radiopacities in $\mathrm{OCP} / \mathrm{Gel}$ disks before implantation in the pilot study; thus, the increase of the radiopacity should stand for the enhancement of mineralization or new bone formation, as previously reported. ${ }^{7-10}$ Although the size of the bone defect tended to

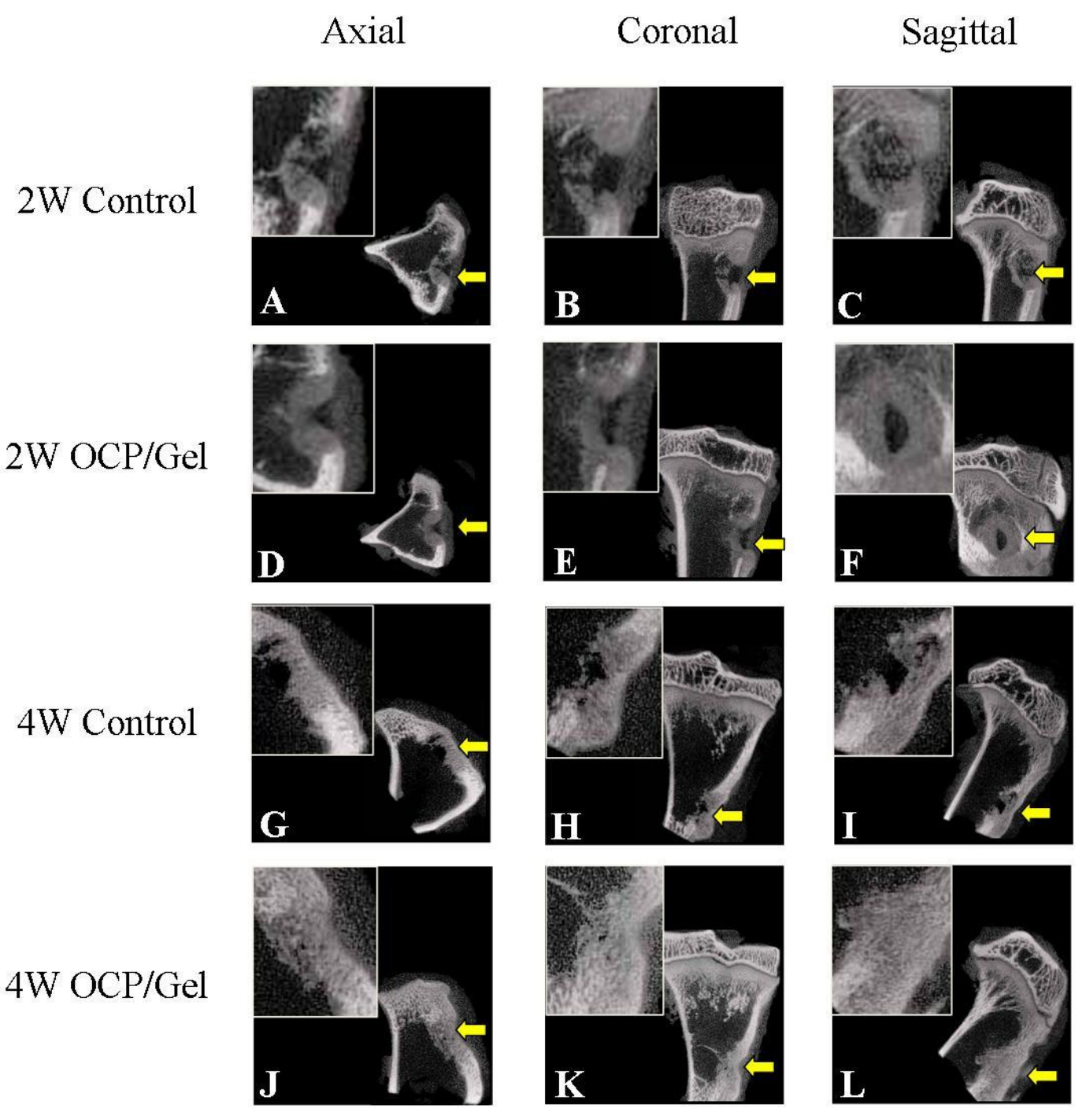

FIGURE 3. Overview and magnified micro-CT images of bone defect regions after treatment with/without $\mathrm{OCP} / \mathrm{Gel}$. The arrows in the overview images represent the magnified area described in the squares. (A, B, C) control, (D, E, F) OCP/Gel at 2 weeks. (G, H, I) control, (J, K, L) OCP/Gel at 4 weeks. (A, D, G, J) axial images, (B, E, H, K) coronal images, (C, F, I, L) sagittal images. 
decrease in both the OCP/Gel-implanted group and the control (defect only) group at 2 weeks, thicker radiopacity was apparent in the $\mathrm{OCP} / \mathrm{Gel}$ group than in the control group. Bone defects were completely restored and continued with bone cortex, even in the bone defect of control at 4 weeks. However, the area repaired was remarkably higher in the OCP/Gel group than in the control group.

\section{3) Histological examination}

The results of histological evaluation at 2 weeks are shown in Figure 4. Although the reactive new bone was formed along the margin of the bone defect on the control groups, connective tissue containing massive fibroblastic cells and several osteoclasts occupied the central part of the bone defect (Fig. 4A, 4C, and 4E). In contrast, the bone defect with OCP/Gel was rapidly bridged by the newly formed bone (Fig. 4B), which may correspond to the increase of the radiopacity caused by the cortical bone deposition. The highly magnified observation disclosed that a structure similar to cartilage is partially discernible around the newly

\section{W Control}
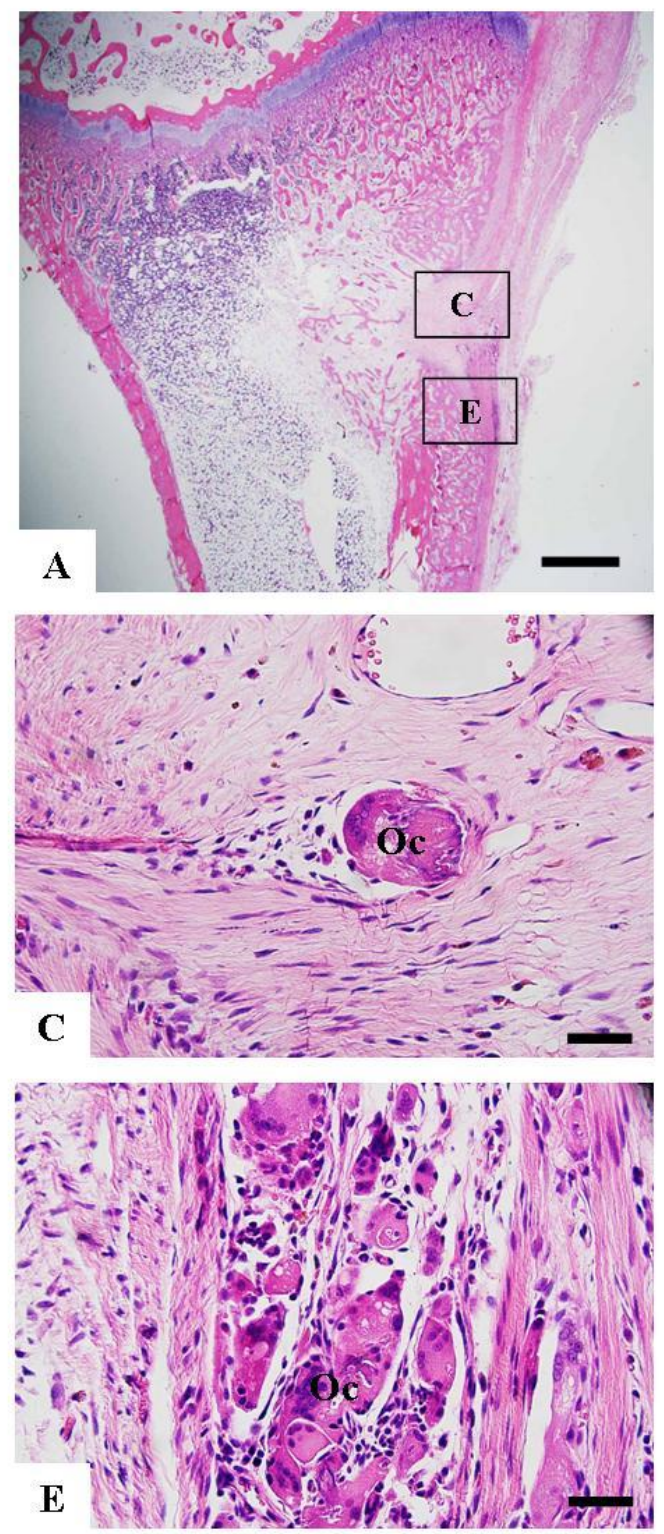

\section{W OCP/Gel}
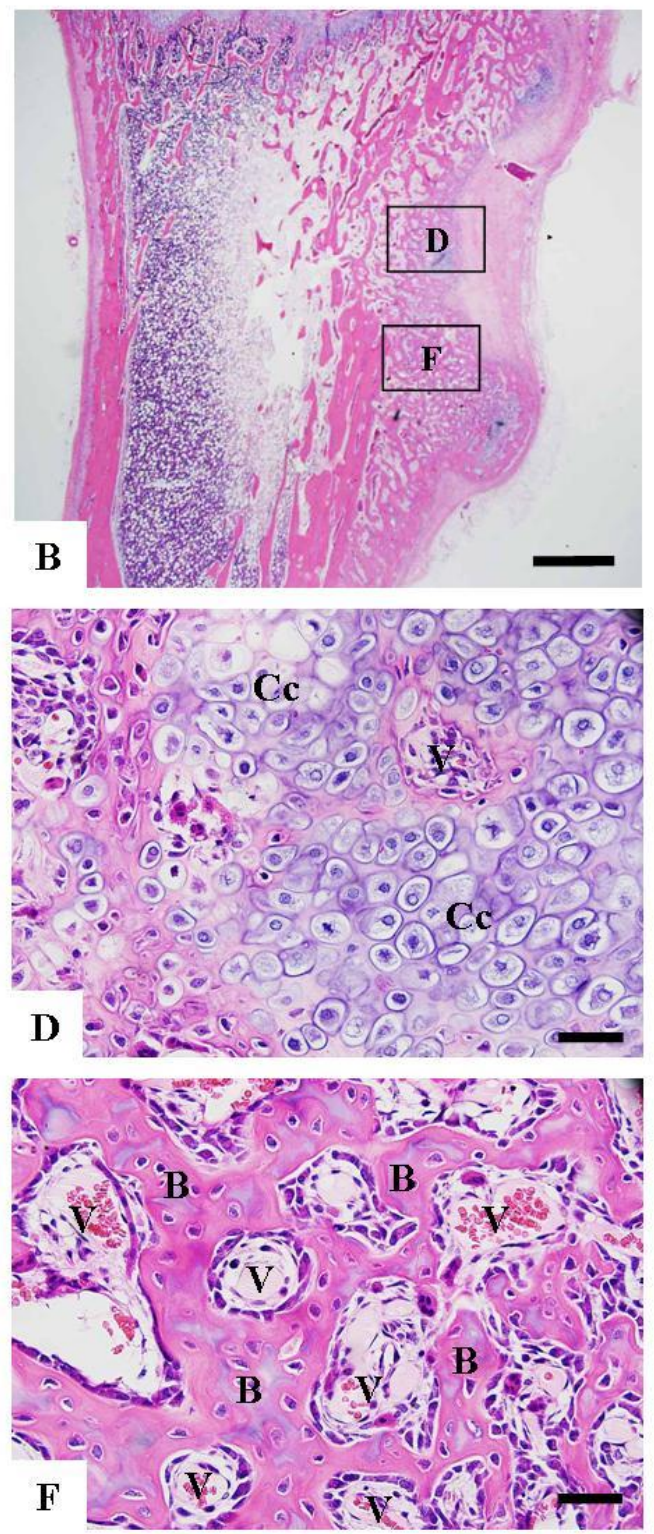

FIGURE 4. Histological micrograph of the control (A, C, E) and OCP/Gel (B, D, F) at 2 weeks post-implantation. The squares represent the magnified area $(C, D, E, F)$ in an overview image (A, B). Oc: osteoclasts. Cc: Chondrocyte. B: Bone matrix. V: Vessel. Bars: $3 \mathrm{~mm}$ for (A, B), $50 \mu \mathrm{m}$ for $(\mathrm{C}, \mathrm{D}, \mathrm{E}, \mathrm{F})$. 
formed bone, suggesting that $\mathrm{OCP} / \mathrm{Gel}$ possibly induces an innate endochondral ossification process in addition to the cortical bone deposition in the defect of the tibia (Fig. 4D). The new bone uniformly constituted a structure similar to bone marrow containing abundant vessels and a bone matrix with embedded cells and osteocytes (Fig. 4F). Light hematoxylinophilic staining in the bone matrix appeared to be a residual of the OCP/Gel (Fig. 4F). ${ }^{10}$

The results of the histological evaluation at 4 weeks are shown in Figure 5. The entire bone defects were occupied by the newly formed bone for both $\mathrm{OCP} / \mathrm{Gel}$ and the control groups. More mature and uniform trabecular bones were formed in the bone defect with OCP/Gel than in the defects of the control groups (Fig. 5C and 5D).

\section{4) Possible mechanism to enhance bone regeneration by OCP/Gel composite}

\section{W Control}

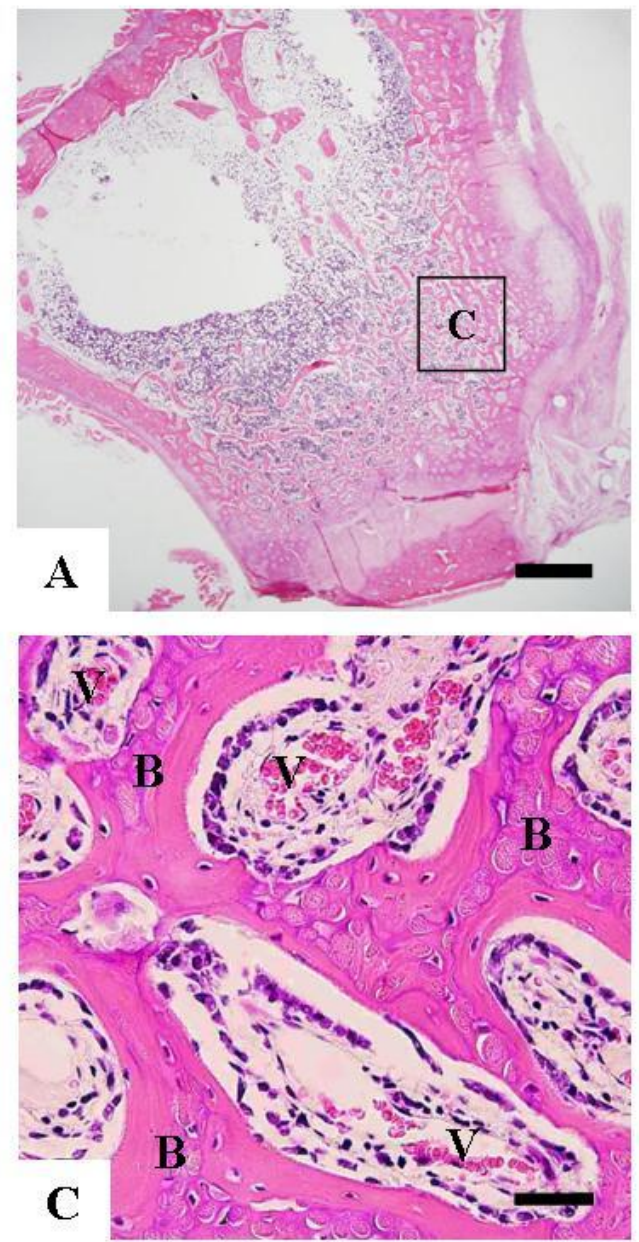

The present study provides evidence that an OCP/Gel composite can more effectively repair a rabbit tibia bone defect more than a control defect in an early stage. We have reported that the OCP/Gel composites have pores in a range of approximately 10 to $500 \mu \mathrm{m}$ and that osteoblastic cells easily migrate into the $\mathrm{OCP} / \mathrm{Gel}$ in vitro, which suggests that host osteoblastic cells can migrate into the materials if implanted. ${ }^{10}$ OCP crystals are capable of enhancing the differentiation of mouse bone marrow stromal cells to osteoblastic cells. ${ }^{1,2,9,13,14}$ OCP enhances osteoblastic cell differentiation and bone regeneration dose-dependently. ${ }^{2,15}$ OCP is a biodegradable material that is mediated by osteoclastic resorption. ${ }^{3,5}$ The OCP/Gel composite has been shown to have a biodegradable characteristic in critically sized rat calvaria. $^{10}$ The present results reconfirm that $\mathrm{OCP} / \mathrm{Gel}$ is a highly biodegradable material if implanted in rabbit tibia. OCP is supposed to be a precursor to biological bone apatite crystals. ${ }^{16}$ The advancement of OCP hydrolysis into HA is

\section{W OCP/Gel}
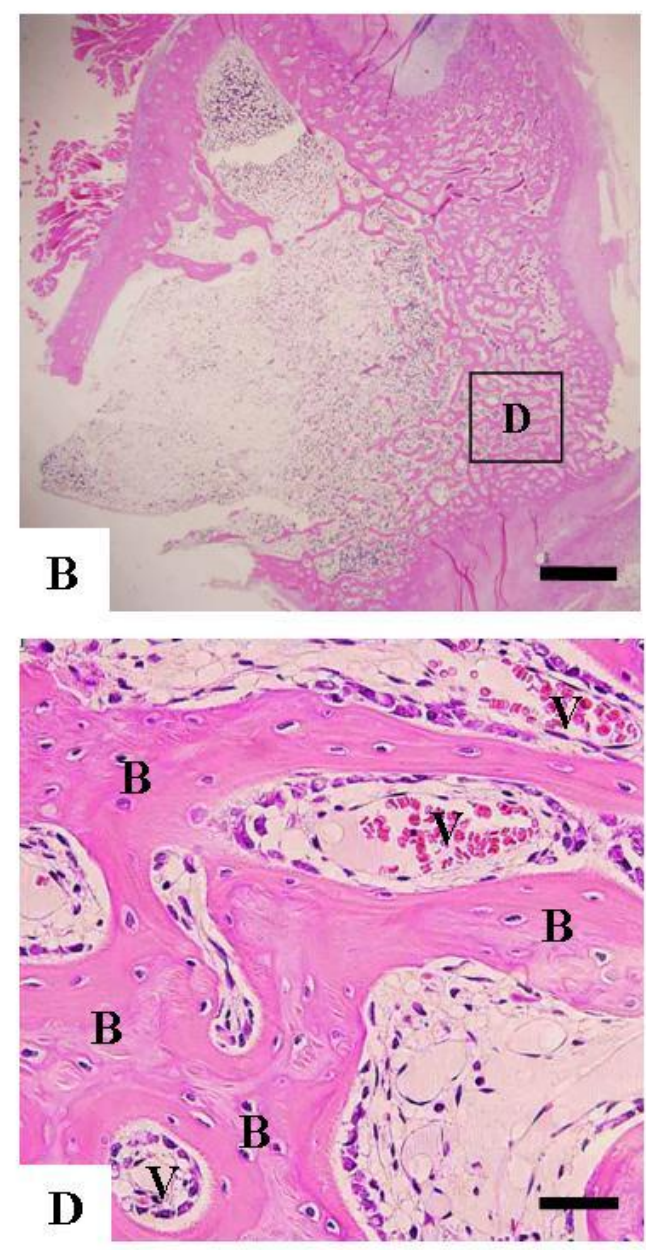

FIGURE 5. Histological micrograph of the control (A, C) and OCP/Gel (B, D) at 4 weeks post-implantation. The squares represent the magnified area $(C, D)$ in an overview image $(A, B)$. B: Bone matrix. V: Vessel. Bars: $3 \mathrm{~mm}$ for $(\mathrm{A}, \mathrm{B})$ and $50 \mu \mathrm{m}$ for $(\mathrm{C}, \mathrm{D})$. 
considered to be associated with the appearance of the stimulatory capacity of the osteoclastic resorption in this mineral salt to enhance bone formation. ${ }^{1}$ The present study suggests that an OCP/Gel composite, utilizing the intrinsic property of OCP to activate osteoblastic and osteoclastic cells, could be used as a bone substitute material in the defect of long bone in the field of orthopedic surgery. Further study is underway to confirm the effect of $\mathrm{OCP} / \mathrm{Gel}$ composite in larger defects that do not repair themselves spontaneously.

\section{CONCLUSION}

An OCP/Gel composite containing approximate $40 \mathrm{wt} \%$ of OCP crystals efficiently repaired the bone defect created in rabbit tibia, coupled with material biodegradation, within 4 weeks. It seems likely that OCP/Gel composite is a scaffold material in which the stimulatory capacity of OCP crystals in bone regeneration can sufficiently be expressed even in the defect of long bone.

\section{ACKNOWLEDGEMENTS}

This study was supported in part by grants in aid (17076001, 19390490, 23390450, 23659909, and 23106010) from the Ministry of Education, Science, Sports, and Culture of Japan and the Suzuken Memorial Foundation.

\section{REFERENCE}

1. O. Suzuki, S. Kamakura, T. Katagiri, M. Nakamura, B. Zhao, Y. Honda, R. Kamijo, Biomaterials, 27, 2671 (2006).

2. T. Anada, T. Kumagai, Y. Honda, T. Masuda, R. Kamijo, S. Kamakura, N. Yoshihara, T. Kuriyagawa, H. Shimauchi, O. Suzuki, Tissue Eng Part A, 14, 965 (2008).

3. M. Takami, A. Mochizuki, A. Yamada, K. Tachi, B. Zhao, Y. Miyamoto, T. Anada, Y. Honda, T. Inoue, M. Nakamura, O. Suzuki, R. Kamijo, Tissue Eng Part A, 15, 3991 (2009).

4. O. Suzuki, M. Nakamura, Y. Miyasaka, M. Kagayama, M. Sakurai, Tohoku J Exp Med, 164, 37 (1991).

5. H. Imaizumi, M. Sakurai, O. Kashimoto, T. Kikawa, O. Suzuki, Calcif Tissue Int, 78, 45 (2006).

6. N. Miyakake, K.N. Kishimoto, T. Anada, H. Imaizumi, E. Itoi, O. Suzuki, Biomaterials, 30, 1005 (2009).

7. S. Kamakura, K. Sasaki, T. Homma, Y. Honda, T. Anada, S. Echigo, O. Suzuki, J Biomed Mater Res B
Appl Biomater, 79, 210 (2006).

8. T. Fuji, T. Anada, Y. Honda, Y. Shiwaku, H. Koike, S. Kamakura, K. Sasaki, O. Suzuki, Tissue Eng Part A, 15, 3525 (2009).

9. T. Kawai, T. Anada, T. Masuda, Y. Honda, Y. Sakai, Y. Kato, S. Kamakura, S. Echigo, O. Suzuki, Eur Cell Mater, 22, 124 (2011).

10. T. Handa, T. Anada, Y. Honda, H. Yamazaki, K. Kobayashi, N. Kanda, S. Kamakura, S. Echigo, O. Suzuki, Acta Biomater, 8, 1190 (2012).

11. A. Tsuchiya, S. Sotome, Y. Asou, M. Kikuchi, Y. Koyama, T . Ogawa, J. Tanaka, K. Shinomiya, J Med Dent Sci, 55, 91 (2008).

12. M. Mathew, W.E. Brown, L. Schoeder, B. Dickens, J Crystallogr Spectrosc Res, 18, 235 (1988).

13. R.M. Shelton, Y. Liu, P.R. Cooper, U. Gbureck, M.J. German, J.E. Barralet, Biomaterials, 27, 2874 (2006).

14. Y. Liu, P.R. Cooper, J.E. Barralet, R.M. Shelton, Biomaterials, 28, 1393 (2007).

15. T. Kawai, T. Anada, Y. Honda, S. Kamakura, K. Matsui, A. Matsui, K. Sasaki, S. Morimoto, S. Echigo, O. Suzuki, Tissue Eng Part A, 15, 23 (2009).

16. W.E. Brown, J.P. Smith, J.R. Lehr, A.W. Frazier, Nature, 196, 1050 (1962). 